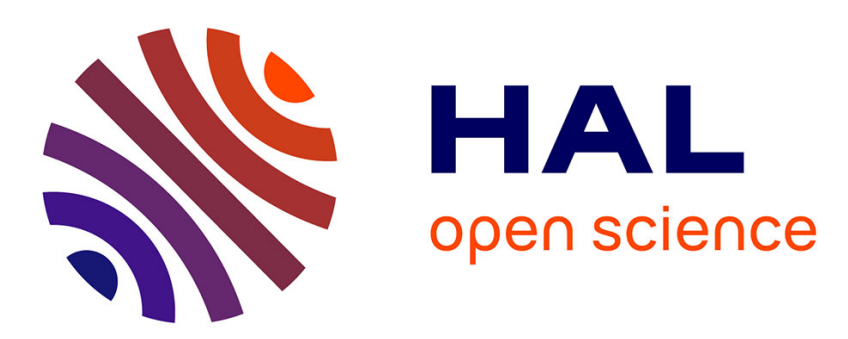

\title{
Étude expérimentale de la lumière émise par un laser à rubis
}

\author{
M. Pauthier, R. Gautier, S. Deiness, G. Amat
}

\section{To cite this version:}

M. Pauthier, R. Gautier, S. Deiness, G. Amat. Étude expérimentale de la lumière émise par un laser à rubis. J. Phys. Radium, 1961, 22 (12), pp.828-832. 10.1051/jphysrad:019610022012082801 . jpa-00236588

\section{HAL Id: jpa-00236588 https://hal.science/jpa-00236588}

Submitted on 1 Jan 1961

HAL is a multi-disciplinary open access archive for the deposit and dissemination of scientific research documents, whether they are published or not. The documents may come from teaching and research institutions in France or abroad, or from public or private research centers.
L'archive ouverte pluridisciplinaire HAL, est destinée au dépôt et à la diffusion de documents scientifiques de niveau recherche, publiés ou non, émanant des établissements d'enseignement et de recherche français ou étrangers, des laboratoires publics ou privés. 


\section{ÉTUDE EXPÉRIMENTALE DE LA LUMIËRE EMISE PAR UN LASER A RUBIS}

Par M. Pauthier, R. Gautier, S. Deiness, et G. Amat

1. Dispositif expérimental. - Avànt d'exposer en détail les observations qui ont pu être faites sur la lumière émise par un laser à rubis, il est souhaitable de donner quelques indications sur le dispositif expérimental qui est d'ailleurs analogue ả celui décrit par Collins et alii (Phys. Rev. Letters, $1^{\text {er }}$ oct. 1960, p. 303). Le rubis est un cristal de corindon $\left(\mathrm{Al}_{2} \mathrm{O}_{3}\right)$ où quelques atomes de chrome se sont substitués à des atomes d'aluminium. Le système de cristallisation est rhomboédrique. Du point de vue optique, c'est un cristal uniaxe. L'indice ordinaire est 1,769, l'indice extraordinaire 1,760 .

Les rubis qui ont été utilisés sont roses, leur concentration est d'environ un atome de chrome pour 2000 atomes d'aluminium. Les atomes de chrome sont en moyenne distants les uns des autres d'environ 30 angströms, ce qui est petit par rapport à la longueur d'onde de la lumière émise ( 7000 angströms).

Les cristaux ont été taillés en cylindres d'environ 40 à $50 \mathrm{~mm}$ de long et 5 à $8 \mathrm{~mm}$ de diamètre. Les deux faces extrêmes sont polies, planes à moins d'une frange près et parallèles entre elles à une fraction de minute près. L'axe du cylindre est, soit parallèle à l'axe optique du cristal, soit perpendiculaire.

Des mesures de diffraction aux rayons $\mathrm{X}$ ont montré que dans les meilleurs échantillons, il peut se produire des variations fortuites d'orientation de l'axe de l'ordre de $1^{\circ}$.

Les deux faces du cylindre sont argentées par évaporation sous vide, l'une de façon à être complètement opaque, l'autre de façon à avoir une transparence d'environ $10 \%$. Le cylindre est ensuite placé au centre

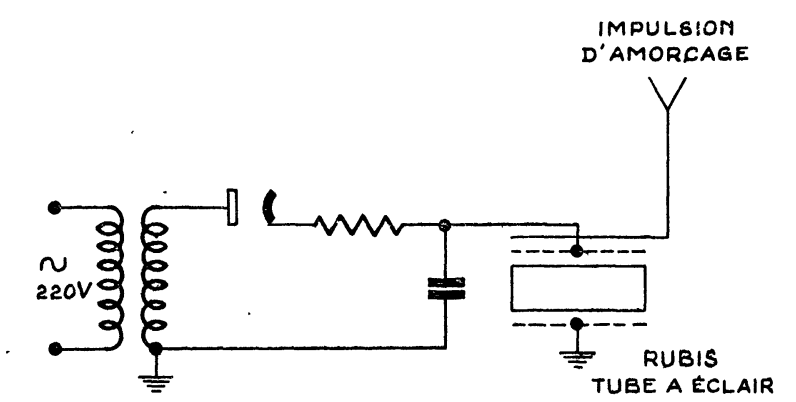

FIG. 1.

d'un tube hélicoïdal en quartz rempli de xénon. On décharge dans ce tube, en l'ionisant au moyen d'une électrode auxiliaire, une batterie de condensateurs suivant le schéma de la figure 1. On observe la lumière 


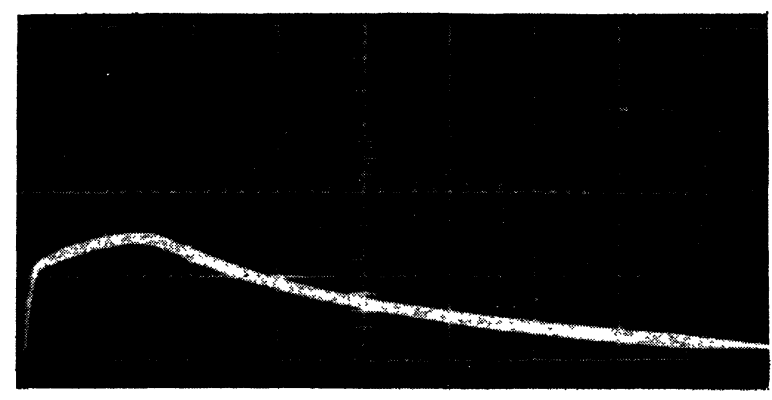

Chiché 1.

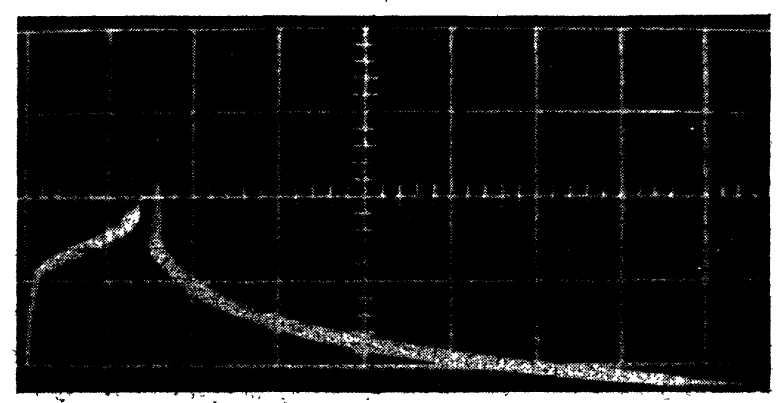

Cutchń 3 .

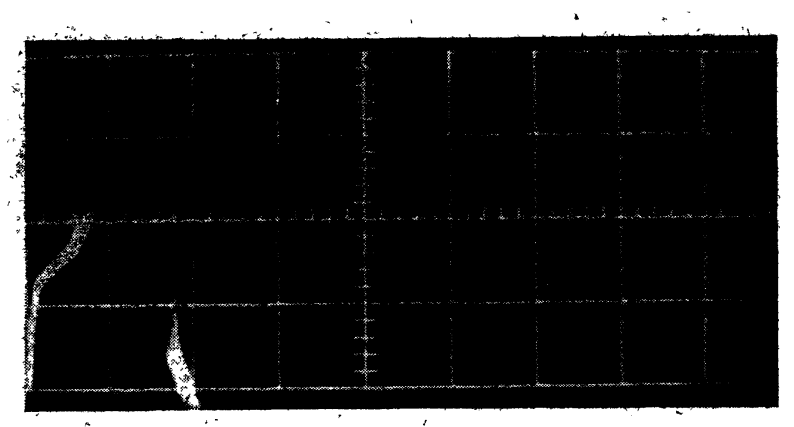

Cú̀ighé 4.

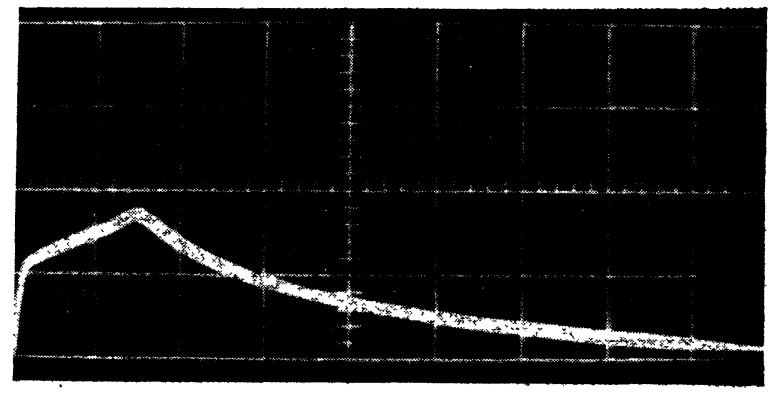

Chíché 2.

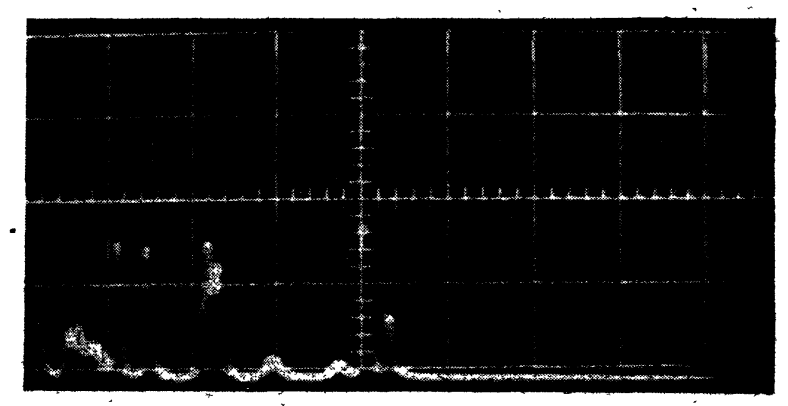

Chuiché 3 A.

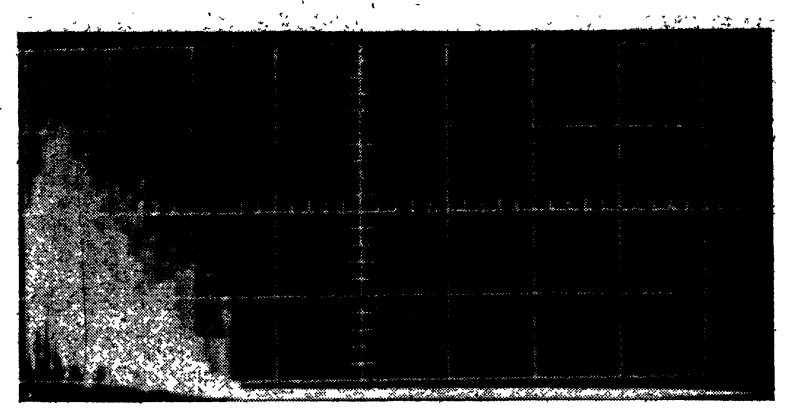

Chiché 4 A.

Fig. 2.

\begin{tabular}{|c|c|c|c|c|c|c|}
\hline CLIChé No & 1 & 2 & 3 & $\begin{array}{c}3 \mathrm{~A} \\
\text { AttÉnUATION } \\
16000\end{array}$ & 4 & $\begin{array}{c}4 \mathrm{~A} \\
\text { ATtÉNUATION } \\
16000\end{array}$ \\
\hline & - & - & - & - & 一 & - \\
\hline $\begin{array}{l}\text { Énergie } \\
\text { de l'éclair } \\
\text { en joules }\end{array}$ & 3100 & 3170 & 3240 & 3240 . & 4000 & 4000 \\
\hline $\begin{array}{l}\text { Échelle } \\
\text { des temps } \\
\text { en } \mathrm{ms} / \mathrm{cm}\end{array}$ & 0,5 & 0,5 & 0,5 & 0,02 & 0,5 & 0,2 \\
\hline
\end{tabular}


émise à travers la face semitransparente du rubis sur un écran placé perpendiculairement à l'axe du cylindre et à environ 2 mètres.

II. Premiers résultats. - Pour de faibles énergies dissipées dans la charge, on n'observe qu'un éclairement uniforme de l'écran dû à la fluorescence du rubis. $\mathrm{Si}$, à capacité constante, on fait croître la tension de décharge, il existe pour chaque rubis et chaque tube à éclair une valeur bien déterminée de cette tension pour laquelle on voit apparaître sur l'écran une tache rouge extrêmement intense d'un diamètre de l'ordre de 10 à $20 \mathrm{~mm}$ suivant les rubis. Si on place un détecteur photoélectrique à l'endroit où apparaît la tache, on peut relever les oscillogrammes suivants placés dans l'ordre des tensions de décharge croissantes (fig. 2).

1. Fluorescence normale du rubis avec décroissance exponentielle qui correspond à une durée de vie moyenne à l'état excité de 4 millisecondes.

2. Au maximum de la fluorescence, une légère pointe apparaît annonçant ainsi le seuil de l'effet laser.

3. Pour une tension supérieure de $1 \%$, l'effet laser se traduit par une très brutale discontinuité de la

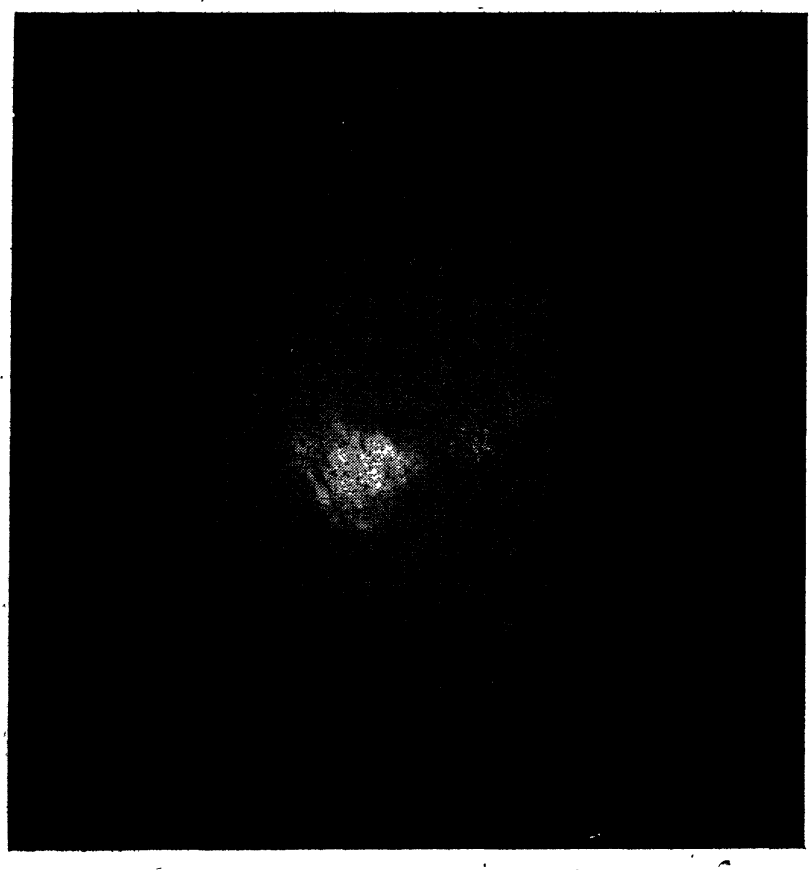

Fig. 3.

seuil, on constate un ensemble de taches distinctes situées de préférence au milieu du rubis ( $f i g .3$ ) et d'un diamètre moyen de quelques dizièmes de millimètres. Lorsque l'énergie de pompage est bien supérieure au seuil, on constate alors qu'il n'y a qu'une grosse tache centrale (fig. 4), mais dont la structure granuleuse laisse penser qu'elle est constituée par la superposition dans le temps et dans l'espace d'une multitude de petites taches élémentaires. Cette supposition rend compte de la directivité relativement faible (de l'ordre courbe. L'ordonnée maximum est des milliers de fois plus grande que le maximum de la fluorescence, ce qui correspond à la concentration de l'énergie lumineuse du rayonnement dans un angle solide très réduit. La fluorescence est encore perceptible après la disparition de l'effet laser, quoique plus faiblement que s'il n'avait pas eu lieu.

Un balayage plus rapide de l'oscilloscope montre que, pendant que se produit l'effet laser, l'énergie est émise sous forme d'impulsions brèves, bien individualisées d'une durée de l'ordre de la microseconde.

4. Pour une énergie de pompage supérieure de $20 \%$ à l'énergie du seuil, la durée et l'intensité de l'effet laser sont fortement accrues. On n'observe alors pratiquement plus de fluorescence après la disparition de l'effet laser.

Un balayage plus rapide de l'oscilloscope montre une forêt d'impulsions se recouvrant les unes les autres.

III. Etude de la face émissive du cristal. - $\mathrm{Au}$ moyen d'un objectif, on peut former l'image agrandie de la face émissive du cristal sur un papier sensible. Lorsque l'énergie de pompage dépasse légèrement le

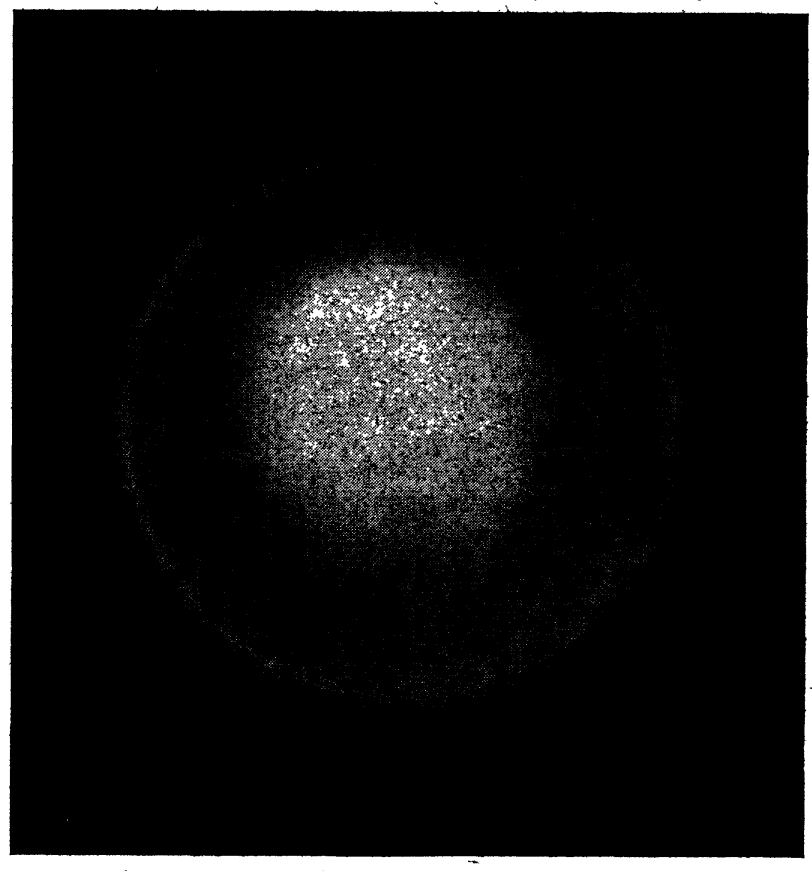

Frg. 4.

du 1/100 de radian) du fäisceau observé. Il est donc possible de considérer le phénomène obtenu comme la superposition au centre du cylindre d'un grand nombre de fibres lasers parallèles à son axe. Si on diaphragme la face du rubis de façon à ne laisser rayonner qu'une petite ouverture de $0,02 \mathrm{~mm}$ de diamètre, l'oscillogramme enregistré montre la présence d'un certain nombre d'impulsions sépàrées, ce qui correspondrait à des oscillations de relaxation dans l'émission d'une fibre. 
IV. Etude du rendement lumineux. - Si on intègre le flux lumineux émis au cours de l'effet laser, on constate que l'énergie lumineuse concentrée dans le faisceau est environ $1 / 2$ joule ( $f g .2$ ). La puissance dissipée dans le tube à éclair peut être très variable suivant la disposition du tube et du rubis. Admettons qu'elle soit de l'ordre de 500 joules. On voit que le rendement est de l'ordre de 1/1000 à peine.

Toutefois, un examen plus serré fait apparaître que ce piètre rendement est principalement dû à l'insuffisance de l'efficacité du dispositif d'illumination, mais que le rubis lui-même a une efficacité tout-à-fait convenable. On peut diviser le rendement total en 4 rendements partiel :

1. Rendement lumineux de la lampe à éclair, c'està-dire rapport de l'énergie lumineuse rayonnée dans la bande spectrale utile à l'énergie électrique totale dissipée, soit $R_{1}$.

2. Rendement géométrique de la lampe, c'est-à-dire rapport du flux lumineux tombant sur le rubis au flux lumineux total rayonné, soit $R_{\mathbf{2}}$.

3. Rendement d'absorption du cristal, c'est-à-dire rapport du nombre d'atomes excités au nombre de photons verts incidents, soit $R_{3}$.

4. Rendements d'émission du cristal, c'est-à-dire rapport de l'énergie en lumière rouge émise en laser à l'énergie absorbée en lumière verte, soit $R_{4}$.

$R_{1}, R_{2}, R_{3}$ sont déterminés par la technologie et susceptibles de grandes améliorations. Leur produit est de l'ordre de $2 \% . R_{4}$ est plus spécialement le rendement laser. Comparons ses valeurs expérimentales et théoriques.

Dans un cylindre de rubis, il y a environ $2.10^{19}$ atomes de chrome. La fréquence de pompage est de $6.10^{14}$ et l'énergie nécessaire au pompage est donc de l'ordre de 8 joules. La fréquence d'émission est $4,3.10^{14}$ et l'énergie maximum qu'on peut penser recueillir est environ 5 joules. On voit donc que le rendement expérimental est de l'ordre de $10 \%$ du maximum théorique, ce qui peut être considéré comme bon pour un dispositif qui est encore en étude dans les laboratoires.

La cause la plus évidente de perte est que seule une fraction inférieure à $50 \%$ de la face du rubis rayonne. Les autres causes sont : l'absorption et la diffusion parasites dues aux défauts du cristal ; les pertes aux réflexions sur les faces latérales et particulièrement la perte à travers la face semi argentée; la fluorescence qui concourt avec l'effet laser pour désexciter les atomes et qui se produit seule tant que le pompage n'a pas atteint la valeur critique.

V. Etude de la polarisation de la lumière émise. Deux cas sont à considérer : barreau taillé perpendiculairement à l'axe optique du cristal et barreau taillé parallèlement. Dans le premier cas (fig. 5), si l'on place devant ce barreau un prisme de Wollaston, on observe pendant l'éclair deux taches.lumineuses sur un écran.En tournant le Wollaston, on obtient l'extinction complète de l'une des deux taches. Pour tous les barreaux qui ont été observés jusqu'ici, la tache qui reste lumineuse correspond à la vibration polarisée rectilignement dont le vecteur électrique est perpendiculaire à l'axe optique.

Én d'autres termes, l'effet laser se produirait préférentiellement, sinon exclusivement, suivant la vibrä- tion ordinaire. Notons que si on émet de la fluorescence pure en déchargeant dans le tube une énergie inférieure au seuil, on s'apervoit que la lumière de fluorescence est partiellement polarisée de la même façon. On peut proposer une explication simple de ce résultat en remarquant que, dans le rubis, l'absorption de la lumière à la longueur d'onde de la fluorescence est moindre pour la vibration ordinaire que pour la vibration extraordinaire.

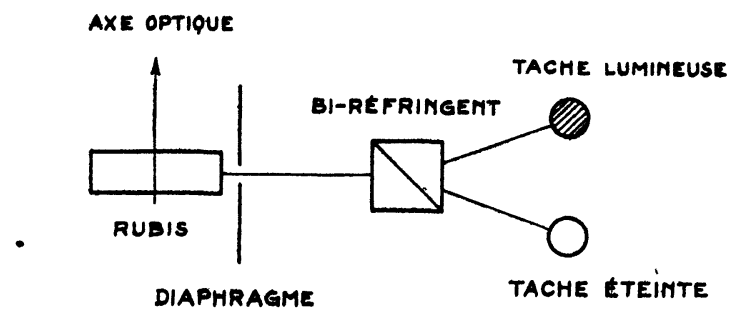

Fig. 5.

Dans la compétition qui s'établit entre les différents modes au départ de l'oscillation maser, ceux qui sont établis sur la vibration extraordinaire sont nettement désavantagés et sont ainsi étouffés par les autres.

Dans le cas maintenant d'un barreau parallèle à l'axe optique du cristal, le montage précédent donne deux taches d'intensité égale, quelle que soit l'orien-

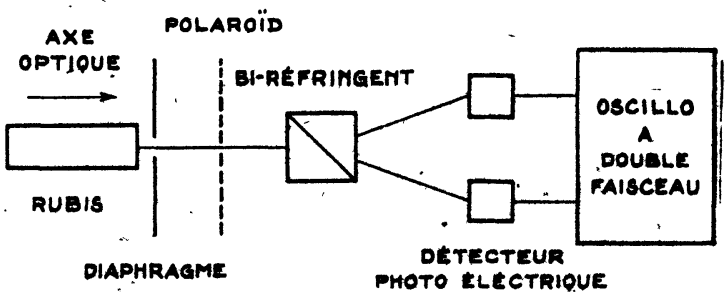

Fig. 6.

tation du Wollaston. L'interposition d'une lame 1/4 d'onde pour la lumière de fluorescence ne change rien au résultat. La lumière n'est donc ni polarisée rectilignement dans son ensemble, ni polarisée circulairement dans son ensemble. On peut penser que chaque impulsion élémentaire qui compose la lumière émise possède unè polarisation déterminée. Il faut donc analyser le phénomène dans le temps (fig. 6). On diaphragme donc la face émettrice du rubis, de façon à n'avoir que quelques impulsions séparées. On place deux cellules photoélectriques à l'emplacement de chacune des taches du montage précédent et on photographie sur un oscilloscope à deux faisceaux l'oscillogramme fourni par chaque cellule. De façon à éviter toute eause d'erreur avant chaque mesure, on fait un essai préalable : on place en avant du Wollaston et à $45^{\circ}$ de ses directions un polaroild. On doit alors obtenir la même courbe sur les deux faisceaux. On retire ensuite le polaroid et on refait un enregistrement.

Les oscillogrammes obtenus sans polaroìd présentent alors des différences notables dans l'amplitude des impulsions homologues, certaines impulsions ayant 
une amplitude quasi nulle sur l'un des faisceaux ( $f$ ig. 7 et fig. 8). Ces résultats semblent bien montrer que la lumière émise reste polarisée d'une certaine façon pendant la durée d'une impulsion. La nature de cette

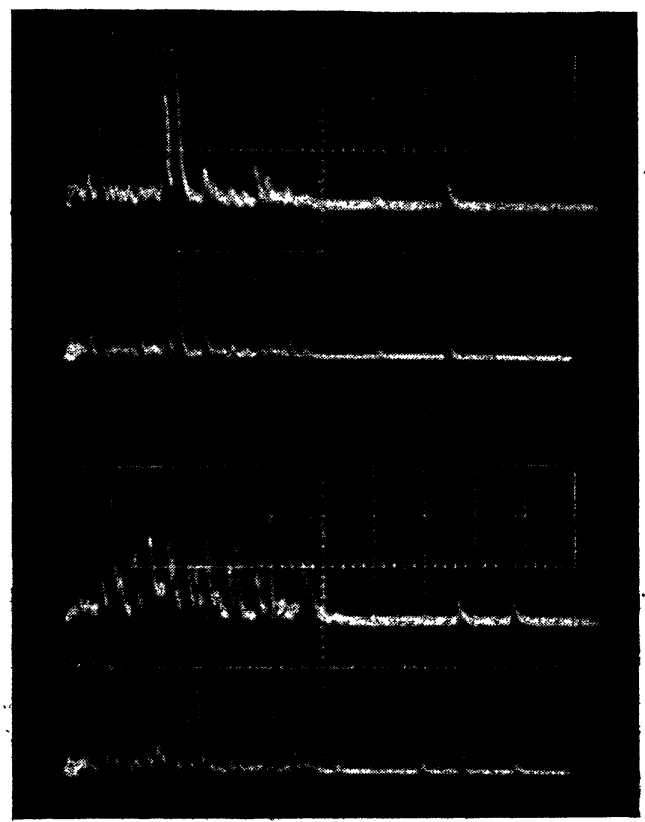

FIG. 7.

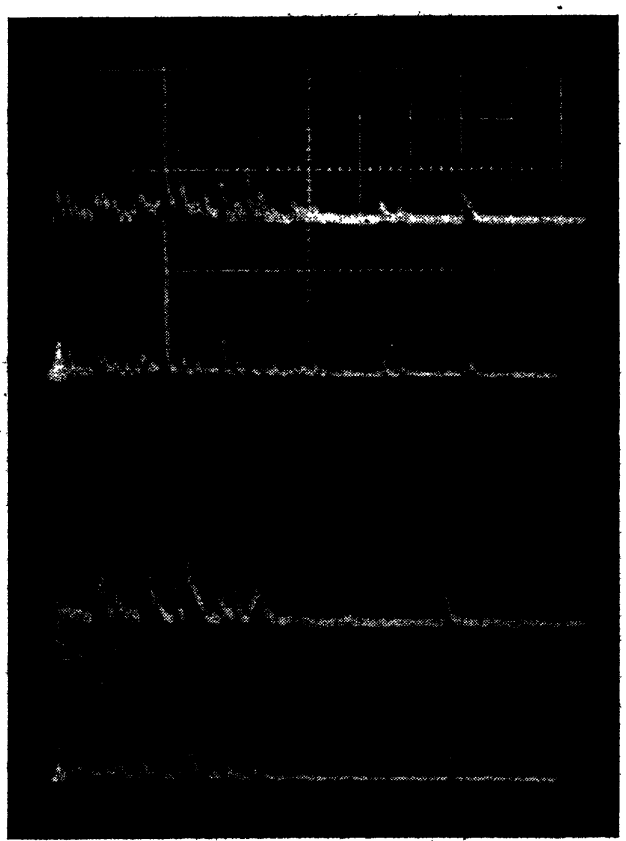

Fig. 8.

polarisation est plus difficile à préciser. Une polarisation rectiligne dont la direction de polarisation serait variable avec chaque impulsion élémentaire ne serait pas incompatible avec ces résultats. M. Kastler nous a fait remarquer qu'une polarisation initialement circulaire à l'intérieur du cristal serait vite déformée si le cristal possédait même une très légère biréfringence dans la direction de propagation. En effet, pour ne provoquer sur un trajet de $5 \mathrm{~cm}$ qu'un retard inférieur au 1/10 de longueur d'onde, il faudrait supposer que la biréfringence est inférieure à $10^{-6}$. Or ceci est bien inférieur à ce que l'on peut pratiquement obtenir à cause par exemple des variations d'orientation de l'axe optique ou des contraintes internes.

VI. Conclusion. - Cette étude expérimentale n'est qu'un début d'investigation. De très nombreux points restent à éclaircir. Le problème expérimental le plus important consiste à démêler les impulsions élémentaires qui composent la lumière émise et à étudier séparément leurs propriétés. Il est probable que ces propriétés se révèleront beaucoup plus près de la cohérence idéale que les propriétés globales de la lumière émise n'ont permis de le montrer jusqu'à présent.

VII. Remerciements. - Les auteurs ont la plus grande reconnaissance pour le Professeur Kastler qui les a guidés bénévolement de ses conseils et leur a prêté du matériel de son laboratoire. Ils remercient très vivement le Laboratoire Central des Télécommunications et, en particulier, M. Goudet qui les a dirigés sur ce travail et les a encouragés et aidés de multiples façons. 